\title{
Coherent shift estimation for stacks of SAR images
}

\author{
Francesco De Zan
}

\begin{abstract}
Speckle tracking is used with SAR images to estimate displacements, in ways that support or integrate interferometric measurements. This paper derives Fisher information expressions for the displacement estimation using coherent speckle tracking, for the multi-image scenario with an arbitrary coherence structure. Previous results were limited to image pairs. An estimator that approaches the theoretical bound is also proposed, establishing a link with phase estimation for multi-image SAR interferometry. The derivation of the Fisher information is given in the general case when the coherence is a function of the frequency.
\end{abstract}

Index Terms-Synthetic Aperture Radar, SAR interferometry, delay estimation, maximum likelihood estimation, covariance matrices

\section{INTRODUCTION}

The most popular technique to measure displacements from SAR images is undoubtedly interferometry. This technique can yield accuracies in the sub-wavelength range, at centrimetric or millimetric level. However there is a second possibility to measure displacements with SAR, which is by maximizing the correlation of two images. There are several incarnations of this technique, depending on the use of complex or amplitude images, and whether the presence of features in the backscatter image is required or not. In this paper I deal with the specific case of coherent speckle tracking, which needs interferometric coherence between the acquisitions, relies on complex signals, but requires no features.

With coherent (and incoherent) speckle tracking the performances are in the order of the resolution element for a few independent samples, that is to say typically much larger than the wavelength and thus much worse than interferometric figures. However we could be interested in this technique for a number of reasons. Coherent tracking is suited for homogeneous areas with no features to track. It will work also in the along-track direction (azimuth), where interferometric tools cannot be applied, because it is a direction orthogonal to range. Moreover, the immunity to phase ambiguity makes speckle tracking useful also in the range direction, where it can support the phase unwrapping problem that inevitably rises in SAR interferometry. This use is detailed in [1] and [2]. Correlation techniques become more and more interesting for geophysical applications as high-resolution SAR systems (in range and/or in azimuth) become progressively available. Examples of coherent speckle tracking can be found in [3] and [4].

The problem of deriving the performance for coherent speckle tracking for SAR images has been already discussed in [5] and [2], together with an efficient estimation scheme.

The author is with the Microwaves and Radar Institute of DLR (German Aerospace Center), P.O. Box 1116, D-82230 Wessling, Germany. email:francesco.dezan@dlr.de
However in those works only the case of two images was investigated, i.e. the estimation of the displacement between two acquisitions. In this paper I shall extend those results to the case of stacks of images, where -generally speakingthe optimal estimation of the shifts requires a dedicated, joint approach. I shall also address the problem of spectral dependency of the coherence.

The original proposal in [1] involved the use of two frequency sub-bands for an efficient shift estimation. Later, many authors have suggested the use of multi-frequency techniques. For example the reader is referred to [6], [7] and [8]. A multifrequency scheme is also found in [9] and [10]. A discussion of the multi-frequency scheme is out of the scope of this letter; the main advantages are related to the reduction of phase wrapping issues along the frequency axis and the possibility to apply spectral weighting in case of non-uniform signal-tonoise ratio.

Ultrasonic imaging applications face similar problems of delay estimation with speckle signals. For example the first results for the two-sensor case can be found in [11]. Developments in the multi-sensor case are described in [12]. However this multi-sensor analysis cannot be identified with the multiimage SAR case and different tools have to be developed.

The first goal of this letter is to present the performance bound relative to the estimation of the shifts in a stack of partially coherent speckle signals. The second goal is to describe an estimation scheme to retrieve those shifts with a performance close to the theoretical bound. Instead of discussing Cramér-Rao bounds I will use an equivalent quantity, which is the Fisher Information.

\section{A. Matricial Notation}

Through this letter bold lower-case symbols are reserved for vectors, upper-case for matrices. Here is some additional notation:

$\mathbf{M}^{T} \quad$ the transpose of a matrix/vector

$\mathbf{M}^{H}$ the transpose-conjugate of a matrix/vector

$\frac{\partial}{\partial \mathbf{y}} \mathbf{x}$

$\odot$

$\delta_{n, k}$

$\mathbf{I}_{N}$

$\mathbf{1}_{N}$

$\mathbf{0}_{N}$

$\mathbf{A}_{(n)}$

$\mathbf{E}_{(n)}$

$[\mathbf{M}]_{n, k}$

$[\mathbf{M}]_{n}$

$\operatorname{Tr}[\mathbf{M}]$ the Jacobian matrix of $\mathbf{x}$ with respect to $\mathbf{y}$ the Hadamard product (element by element) the Kronecker delta the $N \times N$ identity matrix the $N$ element vector $[1,1, \ldots, 1]^{T}$ the $N$ element vector $[0,0, \ldots, 0]^{T}$ a null matrix, except that element $(n, n)$ is 1 a null matrix, except that column $n$ is $\mathbf{1}_{N}$ the element $n, k$ of matrix $\mathbf{M}$ the $n$th column of matrix $\mathbf{M}$ the trace of matrix $\mathbf{M}$ 


\section{PROBLEM FORMULATION}

Let us indicate with $y_{n}(x)$ the $n$th complex speckle signal out of a stack of $N$, with $n=1, \ldots, N$. Each signal will be a delayed version of a corresponding unknown undelayed signal $\xi_{n}(x)$. To fix the ideas one could write

$$
y_{n}(x)=\xi_{n}\left(x-d_{n}\right),
$$

where the $d_{n}$ 's are the various delays or shifts, one per signal or acquisition. These unknown $d_{n}$ 's are the goal of the estimation procedure. Note that the $\xi_{n}(x)$ 's are not identical, albeit aligned to one another. This is because of signal decorrelation, which includes also the effects of additive noise. The coherence properties of the various signals are expressed by the $N \times N$ coherence matrix $\Gamma$ whose elements are:

$$
[\boldsymbol{\Gamma}]_{n, k}=\mathrm{E}\left[\xi_{n}(x) \xi_{k}^{*}(x)\right]
$$

whereas the spectral properties of each signal are given by the autocorrelation function

$$
\begin{aligned}
& \mathrm{E}\left[y_{n}(x) y_{n}^{*}\left(x^{\prime}\right)\right]= \\
& \mathrm{E}\left[\xi_{n}(x) \xi_{n}^{*}\left(x^{\prime}\right)\right]=\operatorname{sinc}\left(x-x^{\prime}\right) .
\end{aligned}
$$

Using the normalized cardinal sine is equivalent to having unitary bandwidth, which simplifies the notation. For the time being let us suppose that there is no dependence of $\boldsymbol{\Gamma}$ on the frequency: this hypothesis will be removed later and a more general result will be presented.

In order to operate with discrete signals I introduce the $N$ vectors $\mathbf{y}_{n}$ which collect the available samples of $y_{n}(x)$, sampled according to Nyquist's limit. For example $x=$ $1,2, \ldots, L$, assuming that $L$ independent samples are available. Explicitely:

$$
\mathbf{y}_{n}=\left[y_{n}(1), y_{n}(2), \ldots, y_{n}(L)\right]
$$

Let us also assume that the vectors $\mathbf{y}_{n}$ are circular-Gaussian distributed, and that the coherence matrix is a complete statistical description (scale factors are obviously irrelevant, so one can safely confuse coherence and covariance matrices).

\section{FISHER INFORMATION MATRIX}

The first result of this paper is that the Fisher information matrix of the vector $\mathbf{d}=\left[d_{1}, \ldots, d_{N}\right]$ given the $\left\{\mathbf{y}_{1}, \mathbf{y}_{2}, \ldots, \mathbf{y}_{N}\right\}$ and for large $L$ is

$$
\mathrm{FIM}_{d}=\left(\boldsymbol{\Gamma} \odot \boldsymbol{\Gamma}^{-1}-\mathbf{I}_{N}\right) 2 L \frac{\pi^{2}}{3} .
$$

Since the signals were scaled to unitary bandwidth in (3), the resulting Fisher information is dimensionless and normalized to the resolution element. The proof is given in the Appendix.

Typically by inverting the Fisher information matrix it is possible to derive the Cramér-Rao covariance bounds of the estimation of the $\mathbf{d}$ [13]. However a precisation is here necessary: the matrix $\mathrm{FIM}_{d}$ is rank deficient and it is thus not invertible. Indeed, using basic properties of the determinant and the inverse matrix, one can show that the vector $\mathbf{1}_{N}$ is always an eigenvector for $\left(\boldsymbol{\Gamma} \odot \boldsymbol{\Gamma}^{-1}-\mathbf{I}_{N}\right)$ with an eigenvalue of 0 . This means that there is no information on any additional delay affecting equally the $d_{n}$. This fact is easily understood considering that the observation of the $\mathbf{y}_{n}$ will carry information concerning the relative shifts between the signals, not the absolute shifts which will stay unknown.

The $\mathrm{FIM}_{d}$ can still be used to find the Cramér-Rao bound on functions of the $\mathbf{d}$, for example the shifts relative to one signal taken as a reference or the average velocity, assuming uniform motion. In general, if one can write $\mathbf{d}$ as a differentiable function of some vector parameter $\boldsymbol{\alpha}$, the corresponding Fisher information can be retrieved through the relation [14]:

$$
\operatorname{FIM}_{\alpha}=\frac{\partial}{\partial \boldsymbol{\alpha}} \mathbf{d}^{\mathbf{T}} \text { FIM }_{\mathbf{d}} \frac{\partial}{\partial \boldsymbol{\alpha}} \mathbf{d}
$$

\section{A. Examples of derived Fisher information}

With two signals $(N=2)$, for example, modelling the shifts as $\mathbf{d}=\left[x_{0}, x_{0}+\Delta x\right]^{T}$ and

$$
\boldsymbol{\Gamma}=\left[\begin{array}{ll}
1 & \gamma \\
\gamma & 1
\end{array}\right], \quad \gamma \in(0,1)
$$

it is possible using (5) and (6) to obtain the information for $\Delta x$, i.e. the relative delay, as

$$
\operatorname{FIM}_{\Delta x}=\frac{\gamma^{2}}{1-\gamma^{2}} 2 L \frac{\pi^{2}}{3},
$$

which is a confirmation of the result derived by other means in [5]. As expected, the Fisher information on $x_{0}$ will instead be zero.

As another example, when the shift history is expected to follow the law of a constant velocity $v$, one could write

$$
\mathbf{d}=v \mathbf{t}+x_{0}, \quad \text { and thus: } \frac{\partial}{\partial v} \mathbf{d}=\mathbf{t},
$$

where $\mathbf{t}$ is the vector collecting the dates of the various acquisitions. Finally, using (5), (6) and (9) the scalar information on $v$ results

$$
\mathrm{FIM}_{v}=\mathbf{t}^{T}\left(\boldsymbol{\Gamma} \odot \boldsymbol{\Gamma}^{-1}-\mathbf{I}_{N}\right) \mathbf{t} 2 L \frac{\pi^{2}}{3} .
$$

An alternative formulation of the same result was published in [15].

In the special case in which the signals share the same speckle component corrupted only by independent additive noise with a given power, it is possible to derive a concise expression for $\operatorname{FIM}_{v}$. For a regular sampling (i.e. $\mathbf{t}=$ $[1,2, \ldots, N])$ the following holds:

$$
\mathrm{FIM}_{v}=\frac{N^{2}\left(N^{2}-1\right) \mathrm{SNR}}{N+\mathrm{SNR}^{-1}} \frac{\pi^{2}}{18} L \simeq \mathrm{SNR} N^{3} \frac{\pi^{2}}{18} L .
$$

In this scenario the signal-to-noise ratio SNR is enough to characterize the whole coherence matrix.

\section{B. Similarity to phase estimation for a stack}

The expression for the $\mathrm{FIM}_{d}$ in (5) is very similar to the Fisher information for interferometric phase estimation in a stack of SAR images presented in [16]. In that work, the Fisher information of a vector $\phi$ of $N$ phases with $L$ independent samples and stack coherence $\Gamma$ was found to be:

$$
\mathrm{FIM}_{\phi}=\left(\boldsymbol{\Gamma} \odot \boldsymbol{\Gamma}^{-1}-\mathbf{I}_{N}\right) 2 L
$$


considering only decorrelation issues (i.e. ignoring atmospheric phase disturbances).

This expression is reported here not only for its similarity to the case under study, but also because I am going to suggest a method to estimate the shifts that relies on the estimation of a set of interferometric phases.

\section{ESTIMATION OF SHIFTS IN A STACK}

This section describes how to implement an estimator for the shifts $\mathbf{d}$ in a stack of images and which performance to expect.

Similar to [1], [2], I propose a "Delta-k" or split-band approach. The rationale of split-band exploits the correspondence between delays in the space/time domain to phase slopes in the frequency domain. It takes the difference of the interferometric phases at two frequency points. In fact it is a double difference method (two images and two frequencies) and it measures a relative delay.

To extend this approach to the multi-image case, I suggest to filter the lower and the upper third of the bandwidth of each signal $\mathbf{y}_{n}$. So doing, one would have a set of $\mathbf{y}_{n}^{\prime}$ and a set of $\mathbf{y}_{n}^{\prime \prime}$ vectors, each counting only $1 / 3$ of the original $L$ independent samples.

From the lower-bandwidth set $\left\{\mathbf{y}_{1}^{\prime}, \mathbf{y}_{2}^{\prime}, \ldots, \mathbf{y}_{N}^{\prime}\right\}$ one would then estimate $N$ phases (actually $N-1$, since one must be taken as a reference), using the algorithm in [16], there called "Phase Linking". It is the maximum likelihood estimator of the phases in a stack, from all the possible $N^{2}$ interferograms that can be formed with the $N$ acquisitions. It requires the knowledge of the coherence matrix $\boldsymbol{\Gamma}$, which will be practically estimated from the data themselves or derived from a model. Let us collect the estimated phases in a $N \times 1$ vector $\phi^{\prime}$. The same procedure will be applied to the higher-bandwidth set $\left\{\mathbf{y}_{1}^{\prime \prime}, \mathbf{y}_{2}^{\prime \prime}, \ldots, \mathbf{y}_{N}^{\prime \prime}\right\}$ to get $\phi^{\prime \prime}$.

Finally one would take the differences of the two vectors, $\Delta \phi=\phi^{\prime \prime}-\phi^{\prime}$, and scale it by $3 /(4 \pi)$, which comes from inverting the basic phase-delay relation

$$
\Delta \phi=2 \pi \Delta f \mathbf{d}
$$

for $\Delta f=2 / 3$, which is the frequency separation between the two sub-bands. Of course phase ambiguities have to be considered in case the delay is larger than $3 / 4$ of the resolution cell.

The described procedure is the natural extension of the split-band principle to a stack. The only difference is that for the two-image case the phase estimation step is trivially averaging the interferogram complex samples, whereas in the multi-image case it requires a more complex optimization (for example the mentioned Phase Linking).

\section{A. Performance of the proposed estimator}

The performance of the proposed estimator will be derived from the bound for phase estimates (12), with proper scaling factors. The first scaling factor is due to the reduced number of independent samples, and it amounts to $1 / 3$. The second is due to the phase-delay relation (13) and is $(4 \pi / 3)^{2}$. The last one is a factor of $1 / 2$ due to the difference $\phi^{\prime \prime}-\phi^{\prime}$.

$$
\begin{aligned}
\mathrm{FIM}_{d}^{\prime} & =\left(\boldsymbol{\Gamma} \odot \boldsymbol{\Gamma}^{-1}-\mathbf{I}_{N}\right) 2 L(1 / 3)(4 \pi / 3)^{2}(1 / 2) \\
& =\left(\boldsymbol{\Gamma} \odot \boldsymbol{\Gamma}^{-1}-\mathbf{I}_{N}\right) 2 L \frac{\pi^{2}}{3}(8 / 9) \\
& =\mathrm{FIM}_{d}(8 / 9)
\end{aligned}
$$

The loss factor of the proposed procedure is thus $8 / 9$, which corresponds to $0.5 \mathrm{~dB}$ and is the same figure for the split-band algorithm on a single pair of images [5]. Since the PhaseLinking step was already shown in [16] to have a performance asymptotically close to the bound, it can be concluded that the whole procedure will have the same property with respect to its own bound (5), with an additional penalty of $0.5 \mathrm{~dB}$.

\section{FRequency DEPENDENCE of COHERENCE}

The assumption that there is no variation of the coherence across the spectrum may not hold in some cases, for example for the azimuth direction because of the antenna pattern. In this case an additional degradation is expected, since the signal quality is worse where it is most useful, i.e. at the edges of the spectrum. In this scenario the optimum estimator will have to weigh each frequency component according to the position in the spectrum and the noise level. Introducing a dependence of the coherence matrix on the angular frequency $(\boldsymbol{\Gamma}=\boldsymbol{\Gamma}(\omega))$, it is possible to obtain an expression for the Fisher information:

$$
\operatorname{FIM}_{d}=\int_{-\pi}^{\pi} \omega^{2}\left(\boldsymbol{\Gamma}(\omega) \odot \boldsymbol{\Gamma}(\omega)^{-1}-\mathbf{I}_{N}\right) L \frac{d \omega}{\pi} .
$$

The reader is referred to the Appendix for a proof.

In the simple case of two images the correlator will take the following form in the frequency domain [17]:

$$
\hat{\Delta x}=\underset{\Delta x}{\arg \max }\left|\int_{-\pi}^{\pi} \tilde{y}_{1}(\omega) \tilde{y}_{2}^{*}(\omega) W(\omega) e^{j \omega \Delta x} d \omega\right| .
$$

This is the expression of a weighted periodogram. For the Maximum Likelihood estimator the weights are [17]

$$
W(\omega)=\frac{[\boldsymbol{\Gamma}(\omega)]_{1,2}}{1-[\boldsymbol{\Gamma}(\omega)]_{1,2}^{2}} .
$$

The signals $\tilde{y}_{n}(\omega)$ are frequency domain equivalent of the $y_{n}(x)$ 's and are assumed to be normalized:

$$
\mathrm{E}\left[\left|\tilde{y}_{n}(\omega)\right|^{2}\right]=1 .
$$

\section{DiscusSiOn OF ADDITIONAL ISSUES}

So far the bound and the estimator have been discussed for 1-D signals, but with SAR images one deals typically with rectangular windows of data. The formulas still hold counting the total number of samples, multiplying the range and the azimuth extensions of the estimation windows, provided that they extend enough in the direction in which we want to estimate the shifts. When dealing with 2-D shifts the given formulas apply to each direction independently, under the hypothesis that the displacement is a rigid translation.

Sometimes, even if coherent SAR images are available, it is advisable to use amplitudes instead of complex signals. For example when the interferometric phase cannot be properly 
compensated before the shift estimation. However we do not have analytical expressions for the case of incoherent speckle tracking, although we know there is a performance degradation compared to the coherent case of $3 \mathrm{~dB}$ or more in the single image-pair case [2].

\section{APPENDIX}

In this appendix I derive the expressions for the Fisher information given in (5) and (15). For each frequency we have $N$ observations that we collect in a single vector

$$
\tilde{\mathbf{y}}(\omega)=\left[\tilde{y}_{1}(\omega), \tilde{y}_{2}(\omega), \ldots, \tilde{y}_{N}(\omega)\right] .
$$

The corresponding covariance matrix is $\mathbf{C}(\omega)$ is:

$$
[\mathbf{C}(\omega)]_{n, k}=[\boldsymbol{\Gamma}(\omega)]_{n, k} e^{-j \omega\left(d_{n}-d_{k}\right)} .
$$

Thanks to the hypothesis of zero-mean circular Gaussian distribution of the vector $\tilde{\mathbf{y}}(\omega)$, it is possible to write the Fisher information in terms of the covariance matrix $\mathbf{C}(\omega)$ and its derivatives with respect to the unknown parameters, i.e. the $d_{n}$ 's [13], [18], [19]:

$$
\begin{aligned}
& {\left[\operatorname{FIM}_{d}(\omega)\right]_{n, k}} \\
& =\operatorname{tr}\left[\mathbf{C}(\omega)^{-1} \frac{\partial}{\partial d_{n}} \mathbf{C}(\omega) \mathbf{C}(\omega)^{-1} \frac{\partial}{\partial d_{k}} \mathbf{C}(\omega)\right] .
\end{aligned}
$$

The covariances and the derivatives will be evaluated for $\mathbf{d}=\mathbf{0}_{N}$. The total Fisher information will be obtained integrating across the spectrum and multiplying by the number of independent samples (in the time or space domain) [18], [19]:

$$
\operatorname{FIM}_{d}=L \int_{-\pi}^{\pi} \operatorname{FIM}_{d}(\omega) \frac{d \omega}{2 \pi}
$$

The derivative of the covariance matrix with respect to the delays is a fundamental ingredient and results:

$$
\frac{\partial}{\partial d_{n}}[\mathbf{C}(\omega)]_{n, k}=-j \omega[\boldsymbol{\Gamma}(\omega)]_{n, k} e^{-j \omega\left(d_{n}-d_{k}\right)},
$$

or the same with the opposite sign if the derivative is taken with respect to $d_{k}$, or zero if it is done with respect to another delay or if $n=k$.

In order to express the derivative in a compact way I use the additional $N \times N$ matrix $\mathbf{E}_{(n)}$, defined above:

$$
\frac{\partial}{\partial d_{n}} \mathbf{C}(\omega)=-j \omega\left(\left(\mathbf{E}_{(n)}-\mathbf{E}_{(n)}^{T}\right) \odot \boldsymbol{\Gamma}(\omega)\right) .
$$

Then I proceed towards building the (21). To simplify the notation I temporarily drop the dependence of $\mathbf{C}$ and $\boldsymbol{\Gamma}$ on $\omega$. The first two matrices at $\mathbf{d}=\mathbf{0}_{N}$ are:

$$
\mathbf{C}^{-1} \frac{\partial}{\partial d_{n}} \mathbf{C}=-j \omega \boldsymbol{\Gamma}^{-1}\left[\left(\left(\mathbf{E}_{(n)}-\mathbf{E}_{(n)}^{T}\right) \odot \boldsymbol{\Gamma}\right)\right]
$$

One term in (25) is easy to compute:

$$
\left[\boldsymbol{\Gamma}^{-1}\left(\mathbf{E}_{(n)} \odot \boldsymbol{\Gamma}\right)\right]=\mathbf{E}_{(n)} \odot \mathbf{E}_{(n)}^{T}=\mathbf{A}_{(n)} .
$$

To second term in (25) results

$$
\left[\boldsymbol{\Gamma}^{-1}\left(\mathbf{E}_{(n)}^{T} \odot \boldsymbol{\Gamma}\right)\right]=\left[\boldsymbol{\Gamma}^{-1}\right]_{n}[\boldsymbol{\Gamma}]_{n}^{T} .
$$

Putting all the pieces of (21) together:

$$
\begin{aligned}
& \mathbf{C}^{-1} \frac{\partial}{\partial d_{n}} \mathbf{C ~ C ~}^{-1} \frac{\partial}{\partial d_{k}} \mathbf{C} \\
&=-\omega^{2}\left(\mathbf{A}_{(n)}-\left[\boldsymbol{\Gamma}^{-1}\right]_{n}[\boldsymbol{\Gamma}]_{n}^{T}\right)\left(\mathbf{A}_{(k)}-\left[\boldsymbol{\Gamma}^{-1}\right]_{k}[\boldsymbol{\Gamma}]_{k}^{T}\right) \\
&=-\omega^{2}\left(\mathbf{A}_{(n)} \mathbf{A}_{(k)}-\mathbf{A}_{(n)}\left[\boldsymbol{\Gamma}^{-1}\right]_{k}[\boldsymbol{\Gamma}]_{k}^{T}\right. \\
&\left.\quad-\quad \mathbf{A}_{(k)}\left[\boldsymbol{\Gamma}^{-1}\right]_{n}[\boldsymbol{\Gamma}]_{n}^{T}+\left[\boldsymbol{\Gamma}^{-1}\right]_{n}[\boldsymbol{\Gamma}]_{n}^{T}\left[\boldsymbol{\Gamma}^{-1}\right]_{k}[\boldsymbol{\Gamma}]_{k}^{T}\right)
\end{aligned}
$$

and finally taking the trace:

$$
\begin{aligned}
& {\left[\operatorname{FIM}_{d}(\omega)\right]_{n, k}=-\omega^{2}\left(\delta_{n, k}-\left[\boldsymbol{\Gamma}^{-1}\right]_{n, k}[\boldsymbol{\Gamma}]_{n, k}\right.} \\
& \left.-\left[\boldsymbol{\Gamma}^{-1}\right]_{n, k}[\boldsymbol{\Gamma}]_{n, k}+\delta_{n, k}\right) .
\end{aligned}
$$

This expression can be conveniently rewritten in matrix form:

$$
\operatorname{FIM}_{d}(\omega)=2 \omega^{2}\left(\boldsymbol{\Gamma}(\omega) \odot \boldsymbol{\Gamma}^{-1}(\omega)-\mathbf{I}_{N}\right) .
$$

Expression (15) follows readily from (22) and (30). Solving the integral (15) for constant $\boldsymbol{\Gamma}$ gives (5).

It is now easy to generalize (15) to a band-pass case that encompasses both interferometry and radargrammetry. The Fisher information for the shifts in the case of a signal with carrier $\omega_{0}=2 \pi f_{0} / B$ ( $B$ being the bandwidth, $f_{0}$ the carrier frequency) results:

$$
\operatorname{FIM}_{d}=\int_{\omega_{0}-\pi}^{\omega_{0}+\pi} \omega^{2}\left(\boldsymbol{\Gamma}(\omega) \odot \boldsymbol{\Gamma}^{-1}(\omega)-\mathbf{I}_{N}\right) L \frac{d \omega}{\pi} .
$$

As in (15) and (5), the information is normalized to the resolution element. Under the hypothesis that there is no frequency dependence of the coherence:

$$
\operatorname{FIM}_{d}=\left(\boldsymbol{\Gamma} \odot \boldsymbol{\Gamma}^{-1}-\mathbf{I}_{N}\right) 2 L\left(\omega_{0}^{2}+\frac{\pi^{2}}{3}\right) .
$$

This expression becomes (5) for $\omega_{0}=0$ and is equivalent to (12) for $\omega_{0}^{2} \gg \pi^{2} / 3$, when the carrier delay brings virtually all the information. In this way we see that interferometry and (coherent) radargrammetry are just two extreme ends of the same continuum.

\section{AKNOWLEDGEMENT}

The author would like to thank S. Tebaldini, G. Krieger, $\mathrm{N}$. Veneziani and the anonymous reviewers for their helpful comments.

\section{REFERENCES}

[1] S. Madsen and H. Zebker, "Topographic mapping using radar interferometry: Processing techniques," Geoscience and Remote Sensing, IEEE Transactions on, vol. 31, pp. 246-256, Jan 1993.

[2] R. Bamler and M. Eineder, "Accuracy of differential shift estimation by correlation and split-bandwidth interferometry for wideband and deltak SAR systems," IEEE Geosci. Remote Sensing Letters, vol. 2, pp. 151-155, Apr 2005

[3] P. Prats, R. Scheiber, A. Reigber, C. Andres, and R. Horn, "Estimation of the Surface Velocity Field of the Aletsch glacier using multibaseline airborne SAR interferometry,' Geoscience and Remote Sensing, IEEE Transactions on, vol. 47, pp. 419-430, Feb 2009.

[4] S. Barbot, Y. Hamiel, and Y. Fialko, "Space geodetic investigation of the coseismic and postseismic deformation due to the $2003 M_{w} 7.2$ Altai earthquake: Implications for the local lithospheric rheology," Journal of Geophysical Research, vol. 113, pp. B03403, Mar 2008.

[5] R. Bamler, "Interferometric stereo radargrammetry: Absolute height determination from ERS-ENVISAT interferograms," Proc. IGARSS 2000, vol. 2, pp. 742-745, Jul 2000. 
[6] R. Scheiber and A. Moreira, "Coregistration of interferometric SAR images using spectral diversity," Geoscience and Remote Sensing, IEEE Transactions on, vol. 38, no. 5, pp. 2179 -2191, Sep 2000.

[7] V. Pascazio and G. Schirinzi, "Estimation of terrain elevation by multifrequency interferometric wide band SAR data," Signal Processing Letters, IEEE, vol. 8, no. 1, pp. 7 -9, Jan 2001.

[8] N. Veneziani, F. Bovenga, and A. Refice, "A wide-band approach to the absolute phase retrieval in SAR interferometry," Multidimensional Syst. Signal Process., vol. 14, pp. 183-205, Jan 2003.

[9] N. Bechor and H. Zebker, "Measuring two-dimensional movements using a single InSAR pair," Geophys. Res. Lett., vol. 33, pp. L16311.1L16311.5, Aug 2006.

[10] H.-S. Jung, J.-S. Won, and S.-W. Kim, "An improvement of the performance of multiple-aperture SAR interferometry (MAI)," Geoscience and Remote Sensing, IEEE Transactions on, vol. 47, pp. 2859 -2869, Aug. 2009.

[11] W. Walker and G. Trahey, "A fundamental limit on delay estimation using partially correlated speckle signals," Ultrasonics, Ferroelectris and Frequency Control, IEEE Transactions on, vol. 42, pp. 301-308, Mar 1995.

[12] R. Kozick and B. Sadler, "Source localization with distributed sensor arrays and partial spatial coherence," Signal Processing, IEEE Transactions on, vol. 52, pp. 601-616, Mar 2004.

[13] S. M. Kay, Fundamentals of Statistical Processing, vol. 1, Prentice Hall, 1993.

[14] H. L. Van Trees, Optimum Array Processing, Wiley, 2002.

[15] F. De Zan, P. Prats, and G. Krieger, "Mission design and peformance for systematic deformation measurements with a spaceborne SAR system," IGARSS 2009, Proceedings of, Nov 2009.

[16] A. Monti Guarnieri and S. Tebaldini, "On the exploitation of target statistics for SAR interferometry applications," Geoscience and Remote Sensing, IEEE Transactions on, vol. 46, pp. 3436-3443, Nov 2008.

[17] A Hero and S. Schwartz, "A new generalized cross correlator," Acoustics, Speech and Signal Processing, IEEE Transactions on, vol. 33, pp. 38-45, Feb 1985.

[18] B. Friedlander, "On the Cramer-Rao bound for time delay and Doppler estimation," Information Theory, IEEE transactions on, vol. 30, no. 3, pp. 575-580, May 1984.

[19] P. Whittle, "The analysis of multiple stationary time series," Journal of the Royal Stat. Society, vol. 15, no. 1, pp. 125-139, 1953. 